\title{
Understanding Virtual Embodiment: a Phenomenological Lens
}

\author{
Simeon Vidolov \\ University of Muenster \\ simeon.vidolov@gmail.com
}

\author{
Ivan Vidolov \\ The Academyof the Ministry of Interior \\ ivan_vidolov@abv.bg
}

\begin{abstract}
The paper develops a phenomenological perspective on virtual interactions, which focuses on the centrality of the human body for developing meaningful engagements and relationships in virtual settings. From such a stance, the paper problematizes the extant perspectives that are being premised either on the physicality of the human body and thereby faceto-face interactions, or on the negligence of the body and its reduction to digital text in virtual interactions. In contrast, by drawing on the work of Merleau-Ponty, this paper sets a middle ground, which emphasizes the relationship between the phenomenal (lived) body, and the objective (image) body, which also constitutes our engagements with others. The findings of the paper, based on an analysis of an in-depth, longitudinal, exclusively-mediated software development relationship, identify the importance of inter-orienting and inter-presencing practices. These practices show that virtual interactions are qualitatively distinct mode of engagement, which is more-than-linguistic and more-than-task-oriented. This perspective of virtual embodiment is valuable for addressing the research contradictions in the area of virtual interactions, and offering important insights to the recent IS discourse on performativity and ontological inseparability.
\end{abstract}

\section{Introduction}

The technological advances and increased pervasiveness of ICTs have afforded the emergence of distributed and virtual forms of organizing and collaborating. The research on such virtualized interactions has evolved from the notion of virtual organisation [1], with its focus on normative aspects [2] to more empirical illustrations of outsourcing and similar inter-organisational collaborations [3]-[5]. More recently, new virtual platforms and affordances such as Second Life, and the use of avatars seem to have re-invigorated the research attention to the 'virtual' [6].

The extant research in the area, however, cannot account for the divergent empirical outcomes [7], [8]. Instead, research has been dominated by polar claims between the superiority of the face-to-face forms of interactions, deemed as the 'authentic' and 'richest' communication medium, and the techno-utopian views, associated with claims such as 'death of distance' and the irrelevance of the human body [9]. In parallel with these research controversies [10], the emergence of new virtual phenomena challenge our understanding of virtual processes. For instance, more recent phenomena such as cyber dating and unmanned aircraft systems (UAV) have suggested that 'technologist' explanations are insufficient to account for the rich inter-subjective interactions that are taking place in virtual settings. In particular, the dominant claims of disembodiment and negligence of the body in virtual contexts can be disputed by findings that drone pilots are suffering from the stress and fatigue of combat at the same, if not higher, level than many units located physically in the war zone [11]. On the other hand, cases of online dating scams illustrate how people can be tricked into developing genuine intimate relationships in virtual contexts even without any visual cues [12]. In addition, the use of avatars for enhancing the repertoire for expressing and developing a sense of presence in a virtual context has also attracted research attention [6]. Furthermore, claims of disembodied existence associated with 'transhumanism' and 'mind uploading' appear to become an important topic to both practitioners and researchers [13].

This paper will build on the phenomenological tradition, and the work of Merleau-Ponty, more specifically, to develop an alternative understanding of virtual interactions. This perspective will be developed and illustrated in relation to an in-depth, longitudinal case study of an exclusively virtualized software development relationship between India and Ireland.

\section{Understanding the literature on virtual interactions}

Our understanding of how virtual teams collaborate appears to be marked by divergent research claims [10], and empirical outcomes (Hinds and Kiesler 2002). These controversies are related to a more holistic understnading of the collaboration process, that is not just being 'informational' i.e. involving information exchange for the needs of organising and coordinating collective goals and tasks, but also 'intersubjective' i.e. involving relational and social bonding aspects [14]. Such a holistic understanding of 
collaboration points to the active role of the human body and suggests that 'collaboration is a body contact sport' [15, p. 57]. In particular, it is suggested that the human body plays important role in common physical context, and that 'bodywork' improves attention, familiarity, awareness, social bonding and group identity [16]. In contrast, the understanding of virtual teamwork seems to be underpinned by the dictum "out of sight - out of mind" [15, p. 63], and being viewed as less companionable and frequent, but more effortful [17]. As a result, virtual collaboration is usually viewed as inferior to the traditional co-located process. Media Richness Theory (MRT) [18], for instance, is one of the most prominent theories to account for the failures of ICT-mediated work processes by pointing to the 'inferiority' of technology, and implicitly embracing the idea of face-to-face superiority. For instance, it is argued that the traditional teams tend to communicate more effectively than their virtual counterparts due to the lower capacity of technological communication media [19]-[21]. As a result, many studies view face-to-face meetings as a panacea to virtual collaboration [22], [23]. The underlying claim of these studies is that the human body serves as a 'display system' that transmits rich, non-verbal cues, and as such it serves as a 'communicative device' [24]. The connection between physicality and successful intersubjective relationships resonates with the taskmedia fit claims [25] that argue that collaborations that require social bonding might not be appropriate for interactions without any face-to-face meetings.

Whereas, the above view is focused on the physicality of the body, there is another perspective, which is more optimistic about the success of virtual collaboration. In particular, this perspective focuses on the linguistic exchanges through which shared meanings develop, and in this way assigns less significance to the nondiscursive aspects of collaboration [26], [27]. A central claim for such studies is that collaboration outcomes are not determined by the 'communication medium', but rather by the organisational context and underlying conditions for engagement. As a result, these authors argue that virtual collaborations can be as successful and productive as co-located ones. For instance Walther and colleagues develop the social information processing perspective, which argues that relationship development (affection, similarity, trust and informality) is viable in virtual context after adjusting to the new communication medium [28], [29]. The core of their argument is that social, emotional and relational information can still be exchanged in virtual contexts by being encoded and decoded in the textual messages [30]. According to this perspective then, the human body is subsumed and reduced to digital text.
The former perspective favours the physicality of the body and as such it is unable to account for successful cases of virtual projects that do not involve face-toface interactions e.g. [31]. Therefore, this perspective rejects the possibility for virtual embodiment and respectively rich intersubjective processes in virtual context. The latter perspective, has an optimistic outlook on the possibility of virtual interactions, but the human body is neglected and instead substituted by a view on virtual disembodiment.

In the following section, an alternative perspective of virtual embodiment will be synthesized drawing on the work of Merleau-Ponty. This perspective offers insights, which set a middle ground between the two extreme positions of physicality and negligence of the body.

\section{Merleau-Ponty on Embodiment: conceptual section}

A central argument in Merleau-Ponty's work [32], [33] is that the human body is not an inert housing of a Cartesian ego, which receives and transmits meaning. He elaborates this critical stance by differentiating between two main positions "intellectualism" and "empiricism", which view the subject as located in consciousness and separated from the world. In particular, the intellectualists hold the view that expression imposes meaning on the world, whereas, the empiricists see the subject as passively receiving meaning from the outside world [34]. Instead, Merleau-Ponty avoids this division by arguing that the body is the "fabric" of the world, and there is no clear distinction between inside and outside, self and world. Thus, Merleau-Ponty [32, p. 269] emphasizes that our interactions with others are not a matter of connecting private perceptual/ expressive worlds, but an 'intertwining' and coconstitution of an 'inter-world' that gives primacy to the social and collective over the individual and isolated. For him, similarly as for Heidegger, we are thrown into this social and material (inter-)world, which is infused with significance that we carry by inhabiting the world with our bodies [33]. This interworld is not strictly one's own, but crosses and intertwines with that of others. It entails that actions interlock and engage in the intervolving of others, which is a matter of orientation to the other, a process that is primarily intercorporeal i.e. embodied.

Merelau-Ponty doesn't equate the body with the physical body. Instead, differentiates between bodyschema or phenomenal body, and body image or 
physical body. The body-schema is a flexible, plastic, systemic form of distributed agency encompassing what takes place within the boundaries of the physical body as well as the entirety of the spatiality of embodied motility [35, p. 376]. As such this phenomenal or motile body serves as a "general medium for having a world" [33, p. 146]. On the other hand, the body image is the body as object, which is generated from a primarily visual apprehension of the body as an external object; or content of intentionality (or noetic) [36]. In his later work Merleau-Ponty [37] elaborates on the development of the body image during the period of child development when the child visually apprehends its body in the mirror, which reveals the complex relationship between the phenomenal and objective body. The distinction between the lived experience, kinesthetic experience or tactile feeling of one's body, and the visual or specular experience of the body can be also seen as a distinction of the body 'for me' and the body 'for the other': "What is true of his own body, for the child, is also true of the other's body. The child himself feels that he is in the other's body, just as he feels himself to be in his visual image" [37, p. 134]. In this way, Merleau-Ponty problematizes the two extremes of physicality and neglect for the body by reducing it to text.

For Merleau-Ponty the phenomenal and objective bodies are neither separate realms nor simplistically connected. They are rather collectively enacted through a medium that can vary from touch and vision. This intertwinement is called recursivity. In our encounters, we form an 'interbody', where we embody the other and the other simultaneously embodies us, and thereby develop a state of 'compresence': "My two hands "coexist" or are "com-present" because they are one single body's hands. The other person appears through an extension of that compresence; he and I are like organs of one single intercorporeality"' 38, p. 168]. The linkage between the phenomenal body and the body image, or the 'body for me' and 'body for the other' is a matter of recursive relationship. Merleau-Ponty illustrates this recursive relationship with our two hands touching each other, an encounter of touching and being touched, in which it is difficult to distinguish the subject from the object. Similarly, in co-located interactions this relationship of recursivity is manifested in the process of seeing and being seen, which constitutes the intercorporeal relationship between actors.

Understanding the human body as an inter-relationship between the phenomenal body and the body image, expands our understanding of the human body beyond the 'physical' or 'individual': "What counts for the orientation of the spectacle is not my body as it in fact is, as a thing in objective space, but as a system of possible actions, a virtual body with its phenomenal 'place' defined by its task and situation. My body is wherever there is something to be done" [33, p. 250].

In this way the work of Merleau-Ponty allows us to overcome the two extremes positions in the literature on virtual interactions. In particular, drawing on his insights, we can recognize that the forms of virtual interactions that are pervasive nowadays present a new type of relationship of reversibility (between the phenomenal body and body image) that is distinct from the one in co-located settings. In other words, virtual interactions introduce a different medium of reversibility from the one of co-located interactions that is based on 'seeing and being seen' This suggests that virtual interactions should be treated as a distinct type of practices that enact the relationship between the phenomenal body and body image in a novel way [39].

Using these insights, we can understand virtual interactions as a qualitatively distinct mode of enacting the relationship between the phenomenal and objective body, which is central to understanding intersubjective relationships in virtual settings. In this way, we avoid reifying the human body into a physical substance or digital text, but rather focus on the practices that produce this new mode of 'intertwining'. In the following sections, these insights will be illustrated in relation to an in-depth longitudinal case.

\section{Methods}

The objective of this study was to identify nonlinguistic aspects that contribute to the development of virtual signification and that illuminate important aspects of virtual embodiment. The exploratory nature of the study leads to adoption of a qualitative case study approach that aims to generate novel insights from the data in an inductive grounded manner [40]. The paper presents an in-depth, qualitative, longitudinal field study of an offshoring relationship between India and Ireland. Accordingly, we adhere to the principles of data collection analysis [41].

\subsection{Case Description}

IndiaSoft: The company was operating in Ireland since 2004 when its CEO Bruce following an employment with TATA consulting in different European countries had relocated in Ireland. Bruce had later partnered up with Sean who played a role of a 
marketing manager and business developer. The focus of the company was offering software development services to small start-up companies and SMEs.

Purple was a small start-up technology company that specialized in offering security solutions to consumers. The company was located in Cork, Ireland and had its small in-house software development team.

Relationship between Purple and IndiaSoft: Purple had signed up a demanding contract with a big UKbased retailer that was about to start selling their security solutions, bundled with some of their products. Central to this service was a CRM solution that can allow the customers to avail of the security service. A major part of this service was the development of a call centre and database module. While, Purple had an inhouse development team comprised of 4 permanent employees, they have decided to outsource the development to an external vendor. The competitive rates offered by IndiaSoft, coupled with its

availability to start immediately, were amongst the main reasons for selecting them over local vendors.

After a quick start in August 2007, the first project was unfolding smoothly and unproblematically. Due to some issues between Purple and their client, the project was stalled for 3 weeks. Later, however, the project resumed and was brought to a conclusion. The success of the first project pleased the senior management of the two companies, and later they met to discuss another project that was of even greater importance to Purple. The commercial aspects of this second project were quickly negotiated and it kicked off in the middle of December 2007. The module that had to be developed during this project was a laptop and mobile security application, and it was considered of higher priority and closer to the core activities of Purple. The Indian team was working throughout the Christmas holidays in order to meet the tight deadline of the project that was set for the middle of January. Soon after the beginning of the project, problems and communication difficulties started. After a difficult to emerge and the project ended in a commercial dispute.

\subsection{Data Collection \& Analysis}

The data collection took place in parallel with the unfolding of the relationship between the two companies and continued with interactions with the two companies beyond the end of their relationship. The main participants from the three locations were interviewed in two rounds of interviews:

\begin{tabular}{|c|c|c|c|}
\hline & Position & Interview & $\begin{array}{l}\text { Inter- } \\
\text { team } \\
\text { Meetings }\end{array}$ \\
\hline Bruce & $\begin{array}{l}\text { CEO } \\
\text { (JaipurSoft) }\end{array}$ & 2 & 2 \\
\hline Paddy & $\begin{array}{l}\text { Project } \\
\text { manager/ } \\
\text { Technology } \\
\text { Lead (Purple) }\end{array}$ & 3 & 1 \\
\hline Sean & $\begin{array}{l}\text { Chief } \\
\text { Marketing } \\
\text { Officer } \\
\text { (JaipurSoft) }\end{array}$ & 4 & 2 \\
\hline Mike & $\begin{array}{l}\text { Project } \\
\text { manager } \\
\text { (JaipurSoft) }\end{array}$ & 3 & \\
\hline Anthony & $\begin{array}{l}\text { Project } \\
\text { Manager for } \\
\text { Purple during } \\
\text { the second } \\
\text { project }\end{array}$ & 1 & \\
\hline TOTAL & & 13 & 2 \\
\hline
\end{tabular}

Table 1 Empirical work

Most of the interviews were conducted through Skype and were recorded. Informal chats and telephone conversations with project participants further provided additional information. Having the Skype Ids of all project participants made easier maintaining frequent interactions with a view of developing a better relationship. Almost two years after the start of the first project, two meetings with the two companies were organized to present aspects of our findings. These meetings were a valuable opportunity for capturing the mature reflections of the key people involved in the projects.

In addition to the formal and informal conversations, all email correspondence and archival data were thoroughly analyzed. This documentary evidence consisted of all the email correspondence (more than 500 emails) exchanged during the two projects, and all project documentation including project plans, consultant reports, financial information and weekly status reports. The examination of these textual and conceptual artefacts offered a more granular perspective on the mundane interactions and practices sustaining the client-vendor relationship, and compensated some of the challenges associated with the digital divide and depth of the investigation [42].

The analytical strategy borrowed heavily from a grounded theory research perspective [40], [43]. Although we decided against explicitly coding the 
data, our concern was to identify key themes therein, and develop them with reference to extant theoretical literatures [44]. The process of data collection and analysis was iterative allowing new themes to emerge. The analysis consisted of multiple readings of the interview transcripts, field notes and project documentation.

\section{Virtual embodiment practices: analysis}

The discrepant project outcomes illuminated some important aspects of virtual interactions and facilitated the application of the work of Merleau-Ponty to identify the practices that enact the successful virtual reversibility. In contrast with linguistic reductionism, the analysis below points to the non-linguistic practices that enact the virtual relationships, and thereby show how virtual embodiment is performed. The analysis is structured around two main dimensions - these are, inter-orienting and inter-presencing.

\subsection{Inter-orienting practices}

Inter-orienting is central to establishing a sense of relational security and mutuality. This process is constituted of non-linguistic but rather gestural practices that are a form of embodied expressivity manifested in the virtual interactions. More specifically, an important part of the interactions that constitute such relational practices involved interorienting through exchanges of symbolic gifts of time and attention. The understanding of the importance of these exchanges, which were not significant as acts of discursive articulations of propositional project content, but rather as acts of expressing presence and readiness to engage, and thereby of care and commitment, were captured and explicated by Sean: "You have to communicate even if you don't have anything to say. You don't wait to deliver something you just communicate with them." (Sean, November 2007).

In contrast with discursive and semantic dialogue that was based on exchanges of purposeful content, the gestural dialogue had a non-discursive and embodied pretext of signaling presence and orientation by offering attention. For instance, Sean who was located in the on-shore centre in Dublin, and essentially saw his role as a relationship liaison, emphasized on his role to sustain and energize the project cooperation on all levels. Such activities seemed to be important for reducing the anxieties caused by the absence of the other in virtual context:

"Mike would have a meeting with Paddy and then I would do separately, I would know that this meeting is taking place, I would contact Paddy afterwards to ensure that everything is ok, that he was happy with the outcome, I would get minutes of the meeting. Then I would contact Mike to make sure that he is happy, to make sure that the client is happy and cooperating" (Sean, November 2007).

Similar to the goal-oriented aspects of these conversations that were developing commitments about tasks and goals through requesting and offering pieces of information, the intercorporeal dimension of the dialogue had a different currency. Namely, by requesting and offering attention through mediated bodily acts, a disposition of care and commitment gradually developed. For instance, being there for the other or orientated towards one's needs and concerns, be it as a distant body, was important for developing a relational armature and mutuality upon which mundane activities could build on. A sense of care and emotional commitment were not an abstract category but were actively developed through such a mediated bodily expressivity.

Requesting and giving time also co-constructed a mutual orientation and disposition in the first project. The gifts of time were expressed in the time allocated to the 'other', and frequently manifested in embodied conduct. For instance, Mike expressed this by speaking very highly of Paddy's dedication, and how much time he had allocated in the beginning of the project by taking him through different aspects of the system. This symbolic gift was later continuously reciprocated by Mike in his timely engagements and responses, but also in conversations by signaling enthusiasm and in being available at short notice:

"Paddy: Hi Mike, are you available to take us, and the call centre guys through the new system today? Sorry for the short notice. The call centre guys are only available at 12 Irish Time. How does that suit you?

Mike: Paddy that should not be an issue... I will be available for the call. Let's do it!! "(Email exchange between Mike and Paddy, 09.12.2007)

Time and attention as the currency of symbolic exchanges through which the intercorporeal dialogue took place were ephemeral and invisible processes, oftentimes, mediated and performed through discursive interactions, and as a result were rarely brought up in conversations during the first project. In an attempt to reflect on the failure of the second project, however, Bruce, talked about the relational dynamic by pointing to the importance of 
time and attention "I started raising the flag: Paddy we need your time and attention" (Bruce, May, 2008). In addition, despite the endeavours to turn around the negative dynamics in the second project through multiple escalations and back-channeling, the senior managers failed to prevent the dissolution of care and concern. Well after the commercial dispute that ultimately ended the relationship between the two companies, Bruce pointed out the lack of sensitivity to the signals of the other party as the key reason for the relational failure: "I think besides all that and all the structures in place, all the different measures in place, one thing that we always should follow is 'see the flags' ...if that doesn't happen doesn't matter how many processes and structures we have put in..." (Bruce, June 2009). This quote suggests that the virtual embodied expressivity manifested in reduced exchanges of time and attention, and, therefore, silences, absences and delays, or more generally 'turning away' was not recognized and attended adequately.

\subsection{Inter-presencing practices}

Inter-presencing refers to the reversibility of interactions, which is of different type in virtual context from that described by Merleau-Ponty in the handshake or the mutual gaze.

While, the apparent tacit link between touching and being touched and seeing and being seen is disrupted in virtual context, we can also see the importance of expressing or signaling and being expressed at or signaled at.

Reversibility in virtual context is a matter of different practices. Maintaining these dialoguing cycles can be also interpreted as a type of expressivity that projected a caring disposition to the other. Importantly, the speed of responding to requests or rhythm of reversibility also contributed to development and maintenance of intersubjective relations. Figure 1 shows a significant variation of response rate (or rhythm of reversibility) across the two projects. In particular, the first project, characterized by relationships of care and commitment, showed a faster response to requests, whereas the reversibility of the dialogue in the second project was much slower and disconnected, spreading longer periods of time. In particular, 8 out of 10 requests or questions were responded within $6.75 \mathrm{~h}$ during the first project, and only less than 3 out of 10 for the same time during the second project.

Figure 1 Response Time Frequency

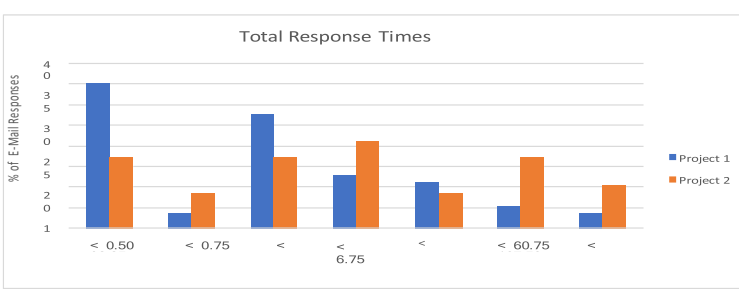

The average response time for the first project was $5 \mathrm{~h}$, while for the second project was $17.9 \mathrm{~h}$. This represents a difference across the two projects of $259 \%$ (Figure 2). It also shows that the distribution of response times is more even during the first project, which also resonates with the relationship of mutuality and care that were partially maintained through the speed of response. In contrast, the big variations in the second project are evidence for silences, absences and delays, which also suggest a breakdown in the co-orientation and cocommitment. These high-level insights also suggested that relationality is a matter of intercorporeal patterns and intertwining rather than of individual acts of signaling.

Figure 2 Distribution of response frequencies

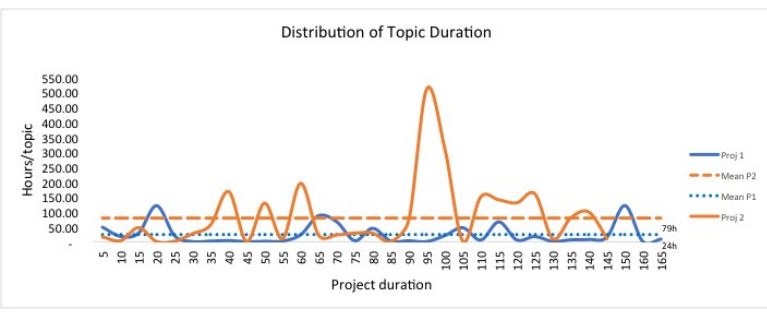

Recognizing the temporal alterity, and in this way heedfully accommodating the 'other' was an important way to show one's care and commitment. This means that the reversibility of the interactions became also a medium for expressivity beyond just projecting presence to the other. A comment by Mike about his experience with another manager of an offshore team further illuminates the importance of reversibility for maintaining and energizing relationships: "He is the kind of phrase 'a real blow buster', you are not waiting for him to get back to you he is back to you before you press the 'send' button so he is really all over us and this really a great contributing factor for the success of the project" (Mike, July 2009). 


\section{Discussion and Conclusion}

This paper opened a new vista for understanding virtual interactions. In particular, it showed that the human body plays a role in intersubjective processes in virtual context. These insights are contrasted with both studies that favour bodily physicality and ones that neglect the role of the human body and advocate notions of virtual disembodiment and linguistic reductionism e.g. [30], [45]. The work of MerleauPonty overcomes this division and suggests that the intertwinement of the phenomenal body and objective body, or of self and 'other' in virtual context is performed in a qualitatively distinct manner.

More specifically, this perspective resonates with recent advances in the IS area that criticize the ontological division of technology from its use, and instead argue in favour of understanding situated technology use as materially enacted practices e.g. [46], [47]. In a similar vein, the work of Merleau-Ponty suggests that the distinct technological medium creates a new intertwinement between the phenomenal and objective body, which is performed through a different set of 'spatializing' or 'materializing' practices. In particular, the paper identifies two types of intercorporeal practices i.e. inter-orienting and interpresencing, which are central to this qualitatively distinct mode of establishing relationships in virtual context. These practices are extra-linguistic and nontask oriented and thus they manifest virtually embodied interactions, which also problematize the emphasis on linguistic exchanges [30].

The findings of the paper add to the few cases of exclusive technology reliance in virtual teamwork [10], and further undermine the ocular-centrism of the studies that view face-to-face meetings as a universal cure to virtual teamwork problems e.g. [22], [23]. Thus, the paper also disputes, the views of virtual disembodiment and instead shows that we should understand virtual interactions as a distinct mode of embodiment [14]. This also suggests that the virtual interactions are not just about technology-mediated activities of the physical body but are rather about a new set of virtual embodied practices, related to the new characteristics of the body image, which is different from the visual or tactile ones.

\subsection{Implications}

The paper offers a number of implications for further study. First, by redefining the rigid division between 'virtual' and 'physical' (or 'real'), the paper problematized claims that oscillate between views of virtual disembodiment or the dissolution of the physical body into a virtual mind. By focusing on the different modes of non-verbal and non-semantic engagements, the study reveals an intercorporeal dynamics, which is constitutive of the collaboration outcomes. This shows how by preserving dualistic orientation about the body, the existing research limits its capacity to develop a holistic understanding of virtual interactions. Such an oversight will preserve the gap between the empirical evidence of successful virtual cases in future studies, and the common conceptual perspectives. Second, this study emphasizes the importance of relational and affective aspects of collaboration, and thus adds to some of the few other authors who consider them in the context of distributed collaboration [48]-[50]. More specifically, this paper doesn't introduce a division between affective or non-task-related, and teleological or goal-oriented aspects of collaboration, but rather shows how they are inter-related [51]. On one hand, the common approach to the process of developing shared understanding misses the relational and affective tonality, and on the other hand, intersubjective relationships develop in the context of common organisational activities. The findings also contribute to attempts to uncover the material and embodied foundation of ephemeral concepts such as social relations or affective atmospheres [52].

This paper also furthers the research debate on virtual relationships by introducing a conceptualization of the virtual interactions as a qualitatively distinct mode of mediated engagement, which refutes claims about virtual disembodiment or reduction of the body to language. The phenomenological sensibility problematizes a reduction of the human body to Newtonian physicality, and the paper illustrated the existential aspects of the body, which is semioticallyextended beyond the immediate physical surrounding, determined by ocular-centrism and visual expressivity. Such a different ontological basis also resonates with the recent turn on 'performativity' and 'sociomateriality', associated with Karen Barad [53], which suggests that the boundaries of 'real' and 'virtual' can be perfromatively reconfigured by situated sociomaterial practices [6].

\section{References}

[1] W. H. Davidow and T. Malone, The Virtual Corporation: Structuring and Revitalizing the 
Corporation for the 21st Century, vol. 2. HarperBusiness, 1992.

[2] L. Introna and B. Tiow, "Thinking about virtual organisations and the future," in 5th European Conference on Information Systems, 1997.

[3] K. M. Chudoba, E. Wynn, M. Lu, and M. B. Watson-Manheim, "How Virtual are we? Measuring and Understanding Its Impact in a Global Organization," Inf. Syst. J., vol. 15, no. 4, pp. 279306, 2005.

[4] S. Faraj, S. L. Jarvenpaa, and A. Majchrzak, "Knowledge Collaboration in Online Communities," Organ. Sci., vol. 22, no. 5, pp. 1224-1239, Oct. 2011.

[5] S. Sarker and S. Sahay, "Implications of space and time for distributed work: an interpretive study of US Norwegian systems development teams," Eur. J. Inf. Syst., vol. 13, pp. 3-20, 2004.

[6] U. Schultze, "Embodiment and presence in virtual worlds: a review," J. Inf. Technol., vol. 25, no. 4, pp. 434-449, Dec. 2010.

[7] L. Introna and M. Brigham, "Derrida, Hospitality and Virtual Community," in Derrida, Business, Ethics, 2008, vol. 44, no. 0, pp. 1-25.

[8] A. Feenberg, "Active and Passive Bodies: Comments on Don Ihde 's Bodies in Technology," Techne, vol. 7, no. 2, pp. 102-109, 2004.

[9] G. Olson and J. Olson, "Distance Matters," HumanComputer Ineractions, vol. 15, pp. 139-178, 2000.

[10] A. Malhotra and A. Majchrzak, "Enhancing performance of geographically distributed teams through targeted use of information and communication technologies," Hum. Relations, vol. 67, no. 4, pp. 389-411, 2014.

[11] N. Gertz, The Philosophy of War and Exile. Palgrave Macmillan, 2014.

[12] T. Buchanan and M. T. Whitty, "The online dating romance scam: causes and consequences of victimhood," Psychol. Crime Law, vol. 20, no. 3, pp. 261-283, Mar. 2013.

[13] M. Hauskeller, "My brain, my mind and I: Some philosophical assummptions of mind-uploading," Int. J. Mach. Conscious., vol. 04, no. 01, pp. 187200, Jun. 2012.

[14] P. Hinds and S. Kiesler, Distributed Work, vol. 47. The MIT Press, 2002.

[15] S. Kiesler and J. Cummings, "What do we know about proximity and distance in work groups? A legacy of research.," in Distributed Work, P. J. Hinds and S. Kiesler, Eds. 2002, pp. 57-80.

[16] B. Nardi and S. Whittaker, "The Place of Face-toFace Communication in Distributed Work," in Distributed Work, P. Hinds and S. Kiesler, Eds. MIT Press, 2002, pp. 83-112.

[17] M. B. O’Leary and M. Mortensen, "Go (Con)figure: Subgroups, Imbalance, and Isolates in Geographically Dispersed Teams," Organ. Sci., vol. 21, no. 1, pp. 115-131, 2010.

[18] R. L. Daft and R. H. Lengel, "Organizational information requirements, Media Richness theory and Structural Design," Manage. Sci., vol. 32, no. 5, pp. $554-571,1986$

[19] S. Bharadwaj and K. Saxena, "Knowledge Management in Global Software Teams," October, vol. 30, no. 4, pp. 65-75, 2005.

[20] J. and K. R. E. Galegher and R. E. Kraut, "Computermediated Communication for Intellectual Teamwork: An Experiment in Group Writing," Inf. Syst. Res., vol. 5, no. 2, pp. 110-138, Jun. 1994.

[21] J. Kotlarsky and I. Oshri, "Social ties, knowledge sharing and successful collaboration in globally distributed system development projects," Eur. J. Inf. Syst., vol. 14, no. 1, pp. 37-48, 2005.

[22] M. L. Maznevski and K. M. Chudoba, "Bridging Space Over Time: Global Virtual Team Dynamics and Effectiveness," Organ. Sci., vol. 11, no. 5, pp. 473-492, 2000.

[23] V. Ramesh and A. Dennis, "The Object Oriented Team: Lessons for Virtual Teams from Global Software Development," Syst. Sci., vol. 00, no. c, pp. 1-10, 2002.

[24] F. Biocca, "The Cyborg's Dilemma: Progressive embodiment in virtual environments," J. Comput. Mediat. Commun., vol. 3, no. 2, pp. 1-18, 1997.

[25] J. E. McGrath, "Time, Interaction and Performance (TIP) A Theory of Groups," Small Gr. Res., vol. 22, no. 2, pp. 147-174, 1991.

[26] P. W. Vlaar, P. van Fenema, and V. Tiwari, "Cocreating Understanding and Value in Distributed Work: How Members of Onsite and Offshore Teams Give, Make, Demand and Break Sense," MIS Q., vol. 32 , no. 2 , pp. 227-255, 2008

[27] O. Ngwenyama and A. Lee, "Communication richness in electronic mail: Critical social theory and the contextuality of meaning," MIS $Q$., vol. 21, no. 2, pp. 145-167, 1997.

[28] J. Walther, "Time effects in computer-mediated groups: Past, present, and future," in Distributed work, P. Hinds and S. Kiesler, Eds. MIT Press, 2002, pp. 235-258.

[29] J. M. Wilson, S. G. Straus, and B. McEvily, "All in due time: The development of trust in computermediated and face-to-face teams," Organ. Behav. Hum. Decis. Process., vol. 99, no. 1, pp. 16-33, 2006.

[30] J. B. Walther, B. Van Der Heide, A. Ramirez, J. K. Burgoon, and J. Peña, "Interpersonal and Hyperpersonal Dimensions of Computer-Mediated Communication," in The Handbook of the Psychology of Communication Technology, S. S. Sundar, Ed. Wiley Blackwell, 2015, pp. 3-22.

[31] A. Malhotra, A. Majchrzak, R. Carman, and V. Lott, "Radical Innovation Without Collocation: A CAse Sudy at Boeing- Rocketdyne," MIS Q., vol. 25, no. 2, pp. 229-249, 2001.

[32] M. Merleau-Ponty, The visible and the invisible. Northwestern University Press, 1969.

[33] M. Merleau-Ponty, Phenomenology of Perception. London: Routledge, 1962.

[34] N. Crossley, Intersubjectivity: The Fabric of Social Becoming. Sage Publicationsc, 1996.

[35] S. Gallagher and J. Cole, "Body image and body 
schema in a deafferented subject," J. Mind Behav., vol. 16, pp. 369-390, 1995.

[36] S. Gallagher, "Body Schema and Intentionality," in The Body and the Self, 1997, pp. 225-244.

[37] M. Merleau-Ponty, "The Child's Relations with Others," in The Primacy of Perception, J. Edie. Evanston, Ed. Northwestern University Press, 1964.

[38] M. Merleau-Ponty, Signs. Northwestern University Press, 1964.

[39] M. Hansen, Bodies in code: interfaces with digital media. Taylor \& Francis Group, 2006.

[40] A. L. Strauss and J. Corbin, Basics of Qualitative Research. Sage Publications, 1990.

[41] H. H. K. Klein and M. Myers, "A set of principles for conducting and evaluating interpretive field studies in information systems," MIS $Q$., vol. 23, no. 1, pp. 67-93, 1999.

[42] G. Marcus, "Ethnography In/Of the World System: the Emergence of Multi-Sited Ethnography," Аnnu. Rev. Anthropol., vol. 24, 1995.

[43] A. L. Strauss and J. Corbin, Grounded Theory in Practice. Sage Publications, 1997.

[44] G. Walsham, "The Emergence of Interpretivism in IS Research," no. 1987, 1995.

[45] J. B. Walther, "Interpersonal Effects in ComputerMediated Interaction: A Relational Perspective," Communic. Res., vol. 19, no. 1, pp. 52-90, 1992.
[46] M. Beane and W. J. Orlikowski, "What Difference Does a Robot Make? The Material Enactment of Distributed Coordination," Organ. Sci., vol. 26, no. 6, pp. 1553-1573, 2015.

[47] L. Introna, "The Enframing of Code Agency, Originality and the Plagiarist," Theory, Cult. Soc., vol. 28, no. 6, pp. 113-141, 2011.

[48] J. Gittell, "New Directions for Relational Coordination theory," in The Oxford Handbook of Positive Organizational Scholarship, G. M. Spreitzer and K. Cameron, Eds. 2010, pp. 400-411.

[49] G. Bendelow and S. Williams, Emotions in Social Life: Critical Themes and Contemporary Issues. Routledge, 1998.

[50] H. Tsoukas, "A Dialogical Approach to the Creation of New Knowledge in Organizations," Organ. Sci., vol. 20, no. 6, pp. 941-957, Dec. 2009.

[51] A. Reckwitz, "Toward a theory of social practices: A development in culturalist theorizing," European Journal of Social Theory, vol. 5, no. 2. pp. 243-263, 2002.

[52] I. Burkitt, Emotions and Social Relations. Sage Publications, 2014.

[53] K. Barad, Meeting the Universe Halfway: quantum physics and the entanglement of matter and meaning. London, Duke University Press., 2007. 